\title{
Which should be treated first; coronary artery disease or celiac artery compression syndrome?
}

\author{
İsmail Bıyık , Erol Bahtiyar², Nilgün Işıksaçan³
}

\footnotetext{
1) Department of Cardiology, Usak University, School of Medicine, Education and Research Hospital, Assoc. Dr., Usak, Turkey

2) Department of Cardiovascular Surgery, Usak University, School of Medicine, Education and Research Hospital, MD., Usak, Turkey

3) Department of Biochemistry, Bakirkoy Sadi Konuk Education and Research Hospital, MD., Istanbul, Turkey
}

\begin{abstract}
Celiac artery compression syndrome is rare. The differential diagnosis and the choice of therapeutic intervention in this syndrome are controversial. Herein, we report of a case of celiac artery compression syndrome and concomitant coronary artery disease and discus differential diagnosis and treatment strategies.
\end{abstract}

Key words: Dunbar syndrome, celiac artery compression syndrome, coroner artery disease, intervention

Bıyık I., Bahtiyar E., Işılksaçan N., Which should be treated first; coronary artery disease or celiac artery compression syndrome? EJCM 2017; 05 (4): 87-90. Doi: 10.15511/ejcm.17.00487. 


\section{Introduction}

Celiac artery compression syndrome (CACS) is a rare clinical entity. It has also known as median arcuate ligament (MAL) or Dunbar syndrome. The disease results from the external compression of the celiac artery by the diaphragmatic crura or the median arcuate ligament. ${ }^{(1)}$ The incidence of this rare condition has been reported from $1.76 \%$ to $4.0 \%$ in different radiologic studies. ${ }^{(1)}$ Approximately, $85 \%$ of patients with CACS are asymptomatic. ${ }^{(1)}$ The leading symptoms of this entity has been reported as abdominal pain (94\%), postprandial abdominal pain ( $80 \%)$, nausea and vomiting (55.6\%), weight loss $(50 \%)$, bloating (39\%), and abdominal pain triggered by exercise $(8 \%) .{ }^{(2)}$ The differential diagnosis and the choice of therapeutic intervention in this syndrome are controversial. ${ }^{(2)}$ Herein, we report of a case of CACS and concomitant coronary artery disease (CAD) and discus differential diagnosis and treatment strategies.

\section{Case Report}

Informed consent of presented patient has been taken for this report. A forty three years old male patient presented to our out-patient clinic of cardiology with the symptoms of long-standing epigastric pain, abdominal swelling and loss of appetite. His detailed history revealed that the symptoms increase especially after overdosed dinner, hiking and exercises. His habitual

Figure 1. Selective coronary angiography

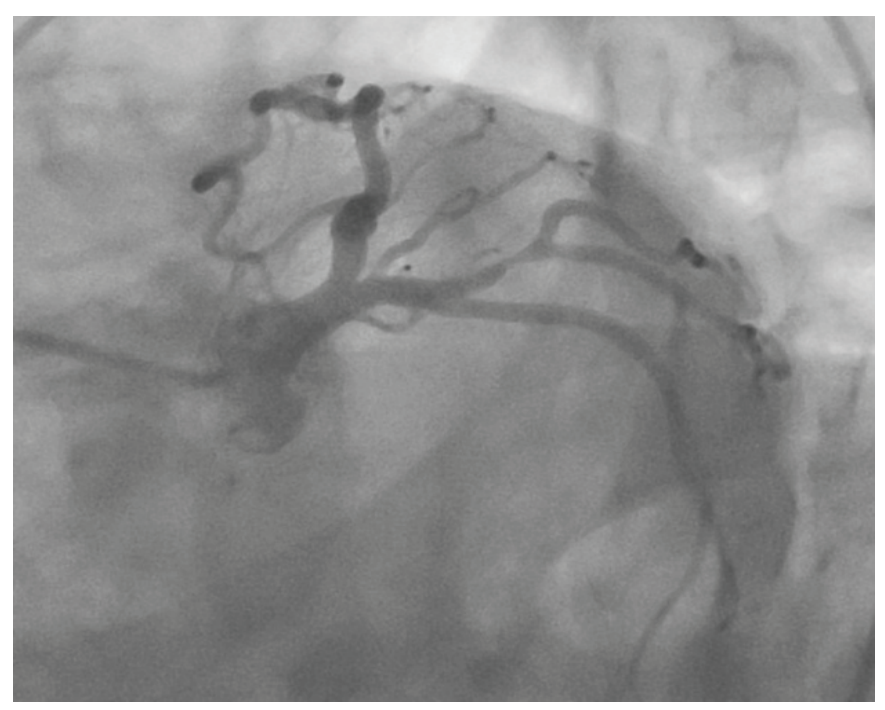

history revealed excessive alcohol and cigarette consumption. His physical examination was unremarkable except abdominal obesity. His body mass index was 32 $\mathrm{kg} / \mathrm{m} 2$. His electrocardiogram and echocardiographic study were normal. His biochemical and laboratory investigations were in normal limits. Exercise electrocardiography showed horizontal ST segment depressions in leads DII, III and AVF at mid-level exercise.

Radionuclide myocardial perfusion scintigraphy also demonstrated reversible inferior wall hypo-perfusion and hypokinesia. Coronary angiography and selective celiac and mesenteric arteriography were performed for the differential diagnosis of symptoms. Coronary angiography displayed critical stenosis visually about 80 to $85 \%$ at the proximal level of well-developed obtuse margin branch of circumflex artery (Figure 1).

In celiac arteriography, there were an external compression on celiac artery causing critical stenosis visually about 80 to $85 \%$ and post-stenotic dilatation of the artery (Figure 2). Computed tomography angiography of abdominal arteries and reconstruction of data confirmed overhead external compression of celiac artery causing critical stenosis and post-stenotic dilatation of the artery (Figure 3). The case was discussed in the council of the cardiology and cardiovascular surgery of the institution. We decided to treat coronary artery disease as first intervention with the stenting of the ob-

Figure 2. Celiac angiography

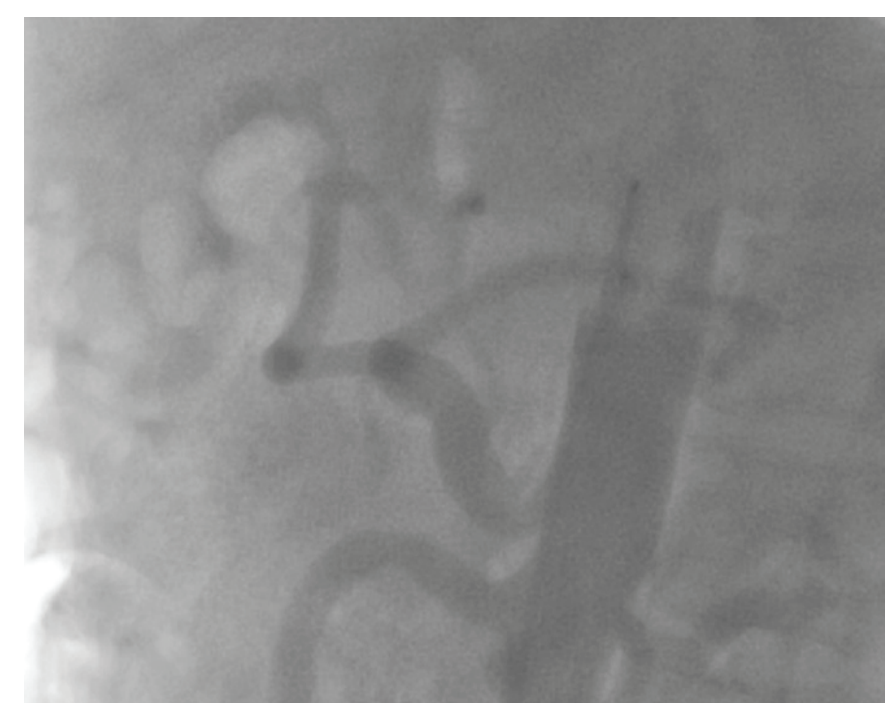


tuse margin branch lesion of circumflex artery and to observe the patient at least one month for the symptoms relief. After successful stenting procedure (Figure 4), the symptoms of the patient quickly relieved, and he was discharged without symptoms. At first month visit, the patient was well condition and there were no his previous complaints.

\section{Discussion}

Symptomatic CACS is a very rare disease, which is clinically determined by the triad of postprandial abdominal pain, weight loss and an abdominal murmur because of the compression of the celiac artery. ${ }^{(3)}$ Fibrous median arcuate ligament usually bestrides the celiac artery at the level of the first lumbar vertebra in $10 \%$ to $24 \%$ of the general population but this entity causes symptoms a few of these patients. ${ }^{(4)}$

There are a lot of reports on this syndrome in literature but its association with coronary artery disease has not been established to date. In the presented case, we took in consideration both CAD and CACS for the source and cause of complaints. Investigative work up was performed in two directions. Celiac arteriography added to coronary angiographic study revealed both critical coronary artery stenosis and celiac artery compression.

Figure 3. Computed tomography angiography of $a b$ dominal arteries and reconstruction of data

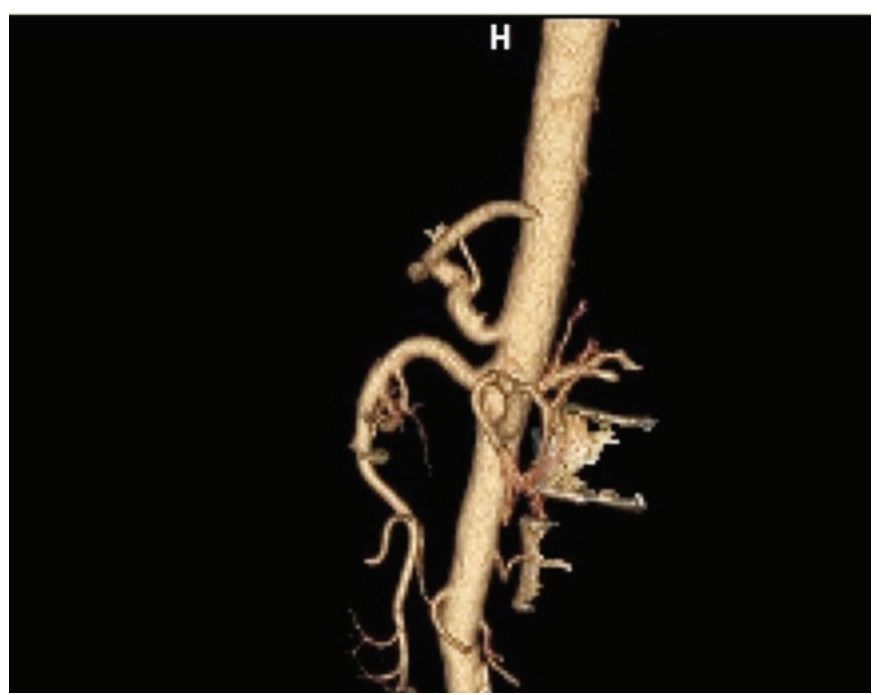

The case was discussed in details in institutional council of cardiology and cardiovascular surgery. Because CACS is frequently encountered but rarely causes symptoms, we decided to treat CAD at first. After successfully performed coronary intervention, patient's complaints and symptoms were disappeared.

CACS could be investigated with Doppler ultrasonography, computed tomography angiography, conventional angiography and magnetic resonance angiography. The traditional method for the treatment of CACS syndrome is open surgical intervention. Recent reports suggest that the laparoscopic surgery is best approach in the treatment of CACS. ${ }^{(5)}$ Laparoscopic MAL release has some advantageous such as decreased postoperative pain, lower incidence of postoperative adhesions, shorter recovery time and hospital stay period. ${ }^{(5)}$ Nowadays, this method has become standard surgical approach for the treatment of CACS. ${ }^{(2)}$

In addition, complex reconstruction procedures such as patch angioplasty, aorta-celiac bypass, reanastomosis of celiac artery to aorta have been performed when needed. ${ }^{(2)}$ In patients having persistent symptoms and residual critical celiac artery stenosis after laparoscopic MAL release, percutaneous endo-

Figure 4. Stenting of obtuse marginal branch of cir cumflex artery

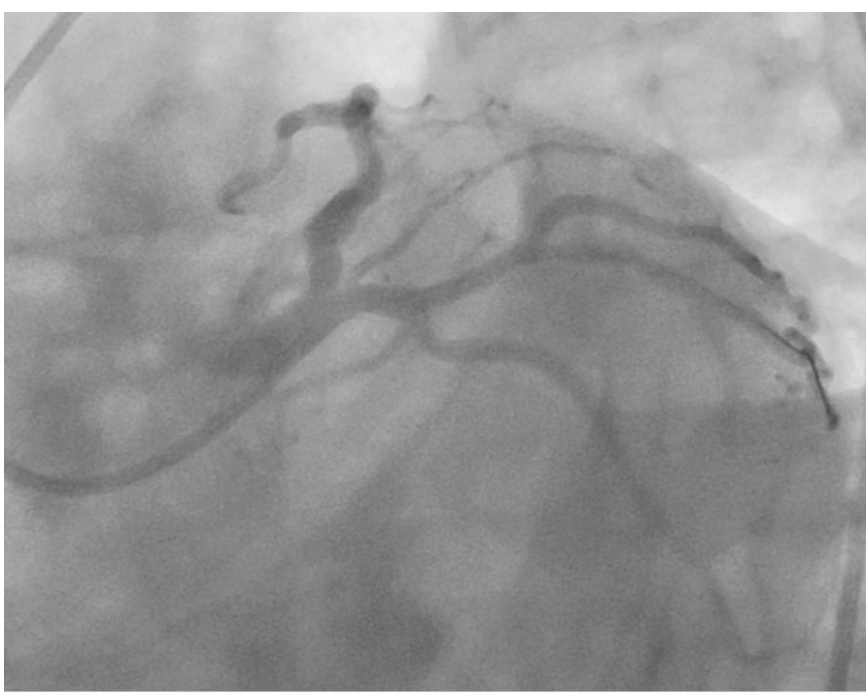

Bıyık I., Bahtiyar E., Işılksaçan N., Which should be treated first; coronary artery disease or celiac artery compression syndrome? EJCM 2017; 05 (4): 87-90. Doi: 10.15511/ejem.17.00487. 
vascular intervention and stenting of persistent critical celiac artery stenosis may be performed. ${ }^{(6)} \mathrm{CAD}$ may mimic the symptoms of CACS especially such as associated with the exercise. Investigational work up should include both diseases.

\section{Conclusion}

Because symptomatic CACS is very rare entity, CAD should be taken into account. Diagnostic work up should include CAD. It may be a reasonable approach to treat coronary artery disease primarily in terms of clinical and prognostic aspects.

\section{References}

1. Gümüş H, Gümüş M, Tekbaş G, Önder H, Ekici F, Çetinçakmak MG, Bilici A. Clinical and multidetector computed tomography findings of patients with median arcuate ligament syndrome. Clin Imaging. 2012; 36(5): 522-5.

2. Kim EN, Lamb K, Relles D, Moudgill N, DiMuzio PJ, Eisenberg JA. Median Arcuate Ligament Syndrome-Review of This Rare Disease. JAMA Surg. 2016; 151(5): 471-7.

3. Kuruvilla A, Murtaza G, Cheema A, Arshad HMS. Median Arcuate Ligament Syndrome: It Is Not Always Gastritis. J Investig Med High Impact
Case Rep. 2017; 5(3): 2324709617728750.

4. Duffy AJ, Panait L, Eisenberg D, Bell RL, Roberts KE, Sumpio B. Management of median arcuate ligament syndrome: a new paradigm. Ann Vasc Surg. 2009; 23: 778-784.

5. Duran M, Simon F, Ertas N, Schelzig H, Floros N. Open vascular treatment of median arcuate ligament syndrome. BMC Surg. 2017; 17(1): 95.

6. Balkan A, Alkan S, Yidirim AE, Konduk BT, Er RE, Gulsen MT. A Rare Cause of Chronic Abdominal Pain: Median Arcuate Ligament Syndrome. Bozok Med J 2017; 7(3): 106-9.

Received: 16/05/2017

Accepted: 21/10/2017

Published: 15/12/2017

Disclosure and conflicts of interest:

Conflicts of interest were not reported.

\section{Corresponding author:}

Dr. İsmail Bıyık

Mail: ismailbiyikmd@yahoo.com 